\title{
Refugees welcome? Active Citizenship und politische Bildungsprozesse durch freiwilliges Engagement
}

\author{
Annette Sprung (D) B Brigitte Kukovetz
}

Eingegangen: 22. März 2018 / Angenommen: 10. Juli 2018 / Online publiziert: 19. Juli 2018

(C) Der/die Autor(en) 2018

Zusammenfassung Freiwilliges Engagement im Bereich von Flucht und Migration stellt einen potenziellen Lernraum dar, in dem vielfältige Kompetenzen erworben werden. Der auf einer empirischen, in Österreich durchgeführten Vorstudie basierende Beitrag analysiert die politischen Lern- und Bildungsprozesse der Unterstützenden unter Berücksichtigung relevanter Einflussfaktoren auf das Engagement, wie z.B. des politischen und medialen Diskurses über Geflüchtete. Im Mittelpunkt der Analyse stehen die Bedeutung von Lernprozessen für die Entfaltung einer aktiven Bürgerschaft sowie die damit verbundenen Herausforderungen für die Erwachsenenbildung.

Schlüsselwörter Freiwilliges Engagement · Flucht · Aktive Bürgerschaft · Politische Bildung · Informelles Lernen

\section{Refugees welcome? Active citizenship and political learning through volunteering}

Abstract Volunteering in the field of migration/refuge opens up a potential learning space for the acquisition of numerous competencies. The paper is based on an empirical pre-study from Austria and focusses on an analysis of political learning

Publisher's Note Springer Nature remains neutral with regard to jurisdictional claims in published maps and institutional affiliations.

Ao. Univ.-Prof. Dr. A. Sprung $(\varangle) \cdot$ Dr. B. Kukovetz

Institut für Erziehungs- und Bildungswissenschaften, Arbeitsbereich

Erwachsenenbildung/Weiterbildung, Karl-Franzens-Universität Graz, Merangasse

70/II, 8010 Graz, Österreich

E-Mail: annette.sprung@uni-graz.at

Dr. B. Kukovetz

E-Mail: Brigitte.Kukovetz@uni-graz.at 
processes amongst volunteers and their implications for the development of active citizenship and the connected challenges for adult education. Diverse factors influencing the engagement of volunteers, such as political and media discourse about refugees, are considered.

Keywords Volunteering - Refugees - Active citizenship · Learning for democracy · Informal learning

\section{Einleitung}

Spätestens seit den umfangreichen Fluchtbewegungen, die in den Jahren 2015 und 2016 die Staaten der europäischen Union (EU) erreichten, dominieren Fragen nach einer asylpolitischen Strategie der EU sowie nach dem konkreten Umgang mit Geflüchteten in den einzelnen Aufnahmeländern vielerorts den politischen Diskurs. Die emotionalisierte Debatte - und damit verbunden kontroverse politische Positionen und Alltagspraxen - reichen von dezidierter Ablehnung bis hin zu solidarischer Unterstützung. Letztere wurde besonders eindrucksvoll im freiwilligen Engagement vieler Menschen sichtbar, die in umfassender Weise zur Bewältigung von Versorgungs- und Inklusionsaufgaben, welche der Staat nicht hinreichend leisten kann oder will -, beitragen (vgl. kritisch dazu van Dyk und Misbach 2016).

Im vorliegenden Beitrag gehen wir der Frage nach, welche politischen Lern- und Bildungsprozesse im Kontext freiwilligen Engagements im genannten Feld stattfinden. Dabei soll zum einen herausgearbeitet werden, was auf der Wissens-, Handlungs- sowie Einstellungsebene gelernt wird. Zum anderen werden Lernkontexte und -settings sowie die damit verbundenen Einflussfaktoren (z. B. medialer Diskurs) beleuchtet. Schließlich sollen die Ergebnisse in Hinblick auf ihre Bedeutung für eine aktive sowie inklusive Bürgerschaft diskutiert werden. Wir sprechen sowohl von Lern- als auch von Bildungsprozessen, da unser Forschungsinteresse u.a. der Frage gilt, inwieweit hier umfassendere Veränderungen im Sinne einer Transformation von Welt- und Selbstverhältnissen der Lernenden vorliegen (vgl. Koller 2012). Unser Blick richtet sich dabei weniger auf die sogenannten Integrationseffekte für Geflüchtete, die ebenso Lern- und Bildungsprozesse vollziehen. Wir nehmen vielmehr das (beobachtbare und potenzielle) Lernen der Aktivistinnen und Aktivisten in den Blick. Es sei jedoch bereits an dieser Stelle angemerkt, dass eine Trennung zwischen Unterstützenden und jenen, denen die Solidarisierung gilt, nicht unbedingt immer so klar zu ziehen ist (vgl. Ünsal 2015).

Wir skizzieren im Folgenden zunächst den Hintergrund der Thematik mit Ausführungen zu Fluchtmigration sowie zum freiwilligen Engagement in Österreich und Deutschland. Diesen beiden Ländern ist gemeinsam, dass sie 2015/16 zu den europäischen Staaten mit den höchsten Flüchtlingszahlen zählten und zu diesem Zeitpunkt auch sehr ähnliche migrationspolitische Rahmenbedingungen und zivilgesellschaftliche Mobilisierungsprozesse aufwiesen. Nach einigen grundsätzlichen Anmerkungen thematisieren wir die Veränderung des öffentlichen Diskurses über Geflüchtete und deren Unterstützerinnen und Unterstützer seit 2015, da - so unsere Hypothese dieser Diskurs einen wichtigen Einflussfaktor auf das Engagement darstellt. Danach 
erfolgt eine detailliertere Betrachtung von Lernprozessen, wie sie in der Forschung zum Lernen in sozialen Bewegungen sowie im freiwilligen Engagement beschrieben werden. Wir verknüpfen diese mit empirischen Ergebnissen aus aktuellen Studien aus Deutschland (Braun 2017; Hamann et al. 2016; Jungk und Morrin 2016; Karakayali und Kleist 2016; Vollmer und Karakayali 2017; Ünsal 2015) sowie mit ausgewählten Ergebnissen einer explorativen Studie (,Solidarität lernen?“), welche wir im Jahr 2016 in Österreich durchgeführt haben und auf der ein im März 2018 gestartetes Forschungsprojekt aufsetzt. Die qualitative Studie orientiert sich an der Grounded Theory Methodologie; bisher wurden sieben leitfadengestützte Interviews mit Freiwilligen - darunter auch in der Koordination/Supervision von Freiwilligen Tätige - rekonstruktiv-interpretativ ausgewertet.

Das freiwillige Engagement analysieren wir schließlich daraufhin, inwiefern hier von aktiver Bürgerschaft bzw. einer sogenannten Inclusive Citizenship gesprochen werden kann.

\section{Flucht und freiwilliges Engagement}

\subsection{Fluchtmigration - aktuelle Entwicklungen}

Der Bericht des Flüchtlings-Hochkommissariats der Vereinten Nationen (UNHCR 2017) weist gegenwärtig einen dramatischen Rekord aus: Mit Ende des Jahres 2016 zählte man weltweit 65,6 Mio. zwangsweise vertriebene Menschen (davon mussten rund 25 Mio. ihre ursprünglichen Heimatländer verlassen). $84 \%$ der Flüchtlinge leben in Entwicklungsregionen. Die meisten Menschen finden derzeit in der Türkei Aufnahme (2,9 Mio.), gefolgt von Pakistan und dem Libanon. Die größte Zahl an Flüchtlingen stammte 2016 aus Syrien, Afghanistan und dem Südsudan (ebd.).

In der EU wurden in den beiden Jahren 2015 und 2016 insgesamt rund 2,5 Mio. Asylanträge gestellt, davon in Deutschland rund 1,16 Mio. und in Österreich ca. 126.000 (Eurostat 2017). Im darauffolgenden Jahr, unter anderem aufgrund restriktiverer politischer Regelungen und Grenzsicherungsmaßnahmen, haben sich die Antragszahlen in den meisten europäischen Staaten jedoch signifikant verringert. Dennoch sind auch für viele Asylwerbende aus den vergangenen Jahren die Bleibeperspektiven bis dato ungeklärt. So befanden sich etwa im Jahr $2017 \mathrm{im}$ Durchschnitt zwischen 79\% und 85\% der Asylwerbenden in Österreich bereits länger als ein Jahr im Verfahren (Fonds Soziales Wien 2017). Eine positive Asylentscheidung erhielten in Deutschland 2016 ca. 25.600 Menschen (BAMF 2017), in Österreich rund 22.300 (BMI 2017). 


\subsection{Freiwilliges Engagement im Kontext von Migration und Flucht}

Als freiwilliges Engagement ${ }^{1}$ wird eine Leistung definiert, „(...) die freiwillig und ohne Bezahlung von Personen außerhalb des eigenen Haushalts erbracht wird, inklusive Maßnahmen zur persönlichen und fachlichen Aus- und Fortbildung für diese Aktivitäten“ (Hofer 2015, S. 3). Als im Spätsommer 2015 hunderttausende Menschen innerhalb eines kurzen Zeitraumes die Grenzen zu Österreich und Deutschland überschritten, wurde deutlich, dass eine staatliche Versorgung der Geflüchteten nicht ausreichend gegeben war. Mediale Berichte über teilweise chaotische Zustände an Grenzen, Bahnhöfen oder im österreichischen Erstaufnahmelager Traiskirchen, aber auch Bilder von Toten auf den Fluchtrouten lösten eine enorme Welle an Empathie und Hilfsbereitschaft der so genannten Zivilgesellschaft aus. Während die akute Notwendigkeit der Hilfe bei der Erstversorgung nach einigen Wochen wieder abebbte, engagierten sich viele Menschen auch weiterhin und längerfristig in der Unterstützung Geflüchteter. Ein nicht unwesentlicher Teil des Engagements besteht seither neben Übersetzungs-, Beratungs- und Begleitdiensten in vielfältigen Bildungsaktivitäten (Spracherwerb, Basisbildung, interkulturelle Begegnungsformate u. v.m.). Diese Engagementformen sind häufig helfend ausgerichtet (und weisen dabei zuweilen einen paternalistischen Gestus auf), während andere einen dezidierten politischen Anspruch verfolgen (auch hier ist Paternalismus nicht auszuschließen) oder gemeinsames solidarisches Handeln zwischen Freiwilligen und Geflüchteten in den Mittelpunkt stellen. Selbstverständlich existieren dazu auch alle möglichen Mischformen. Stärker politisch ausgerichtete Aktivitäten wollen eine breitere Öffentlichkeit für die Lage der betroffenen Gruppe bzw. für Missstände im Asylwesen sensibilisieren (z. B. mit der Unterstützung selbstorganisierter Refugee Protest Camps oder der Veranstaltung von Vorträgen, Kundgebungen, u.ä.). Die Ausprägungen des Engagements variieren stark, ferner verändern sich solidarische Beziehungen oft über die Zeit und es finden folglich unterschiedliche, auch wechselseitige Lernprozesse statt.

Statistische Daten, wie sie im Freiwilligensurvey für Deutschland und im Freiwilligenbericht für Österreich regelmäßig erhoben werden, geben Auskunft über das Gesamtausmaß des freiwilligen Engagements in allen gesellschaftlichen Bereichen (ca. $45 \%$ der erwachsenen Bevölkerung in beiden Ländern) sowie über sozidemographische Merkmale bzw. Motive von Freiwilligen (Hofer 2015; Simonson et al. 2014). Ein allgemeiner Trend hin zu flexiblen, zeitlich begrenzten und projektorientierten Arten der Freiwilligenarbeit ist festzustellen (Zimmer und Vilain 2005, S. 114f.) - dies spiegelt sich ebenso in der Vielzahl der selbstorganisierten Initiativen wider, die im Rahmen der Fluchtbewegungen der jüngsten Vergangenheit entstanden sind.

Erkenntnisse über jene Personen, die sich konkret im Bereich Flucht und Migration engagieren, liegen aus einigen neueren Studien vor, die sich jedoch hauptsächlich auf Deutschland beziehen. Eine (repräsentative) Befragung des sozialwissenschaftli-

\footnotetext{
${ }^{1}$ In der Literatur werden ferner die Begriffe Ehrenamt sowie zivilgesellschaftliches oder bürgerschaftliches Engagement verwendet. Sie sind definitorisch nicht eindeutig voneinander abgegrenzt, werden teilweise synonym gebraucht, haben aber etwas unterschiedliche Konnotationen (etwa in Bezug auf eine eher sozial unterstützende oder politische Tätigkeit).
} 
chen Instituts der Evangelischen Kirche in Deutschland zeigt, dass sich im November 2015 10,9\% und im Mai 2016 11,9\% der Deutschen für Geflüchtete engagiert haben. Zieht man den Einsatz in Form von Geld- und Sachspenden ab, liegt die Quote bei 7,3 bzw. 8,7\% (Ahrens 2017, S. 7). Karakayali und Kleist (2016) haben in wiederholten Befragungen 2014 und 2015² die ,Strukturen und Motive der ehrenamtlichen Flüchtlingsarbeit in Deutschland" analysiert. Sie fanden u.a. heraus, dass drei Viertel aller Engagierten weiblich sind, bei den unter 50-Jährigen liegt dieser Anteil sogar über 80\%. Zwischen 2014 und 2015 hatte der Anteil der 40- bis 60-Jährigen gegenüber den jüngeren Engagierten zugenommen (Karakayali und Kleist 2016, S. 13). Die Freiwilligen sind überdurchschnittlich gebildet und ökonomisch gut abgesichert. Ein hoher Anteil, nämlich 24\% der Befragten, hat selbst einen Migrationshintergrund. Die politische Dimension des Engagements war jenen Befragten, die sich schon vor 2015 freiwillig betätigt hatten, wichtiger als den neuen Akteuren und Akteurinnen (ebd., S. 21). Einen persönlichen Nutzen des Engagements erwarteten sich weniger Menschen als in anderen Bereichen der Freiwilligenarbeit, wo man sich etwa häufig den Erwerb von Qualifikationen erhofft (ebd., S. 30).

Ein wesentlicher Motivationsfaktor war für viele Befragte, v. a. für neu Eingestiegene, ein Gemeinschaftsgefühl mit anderen Freiwilligen zu teilen. 67,4\% nannten ihr Interesse an Menschen aus anderen Kulturen als einen wesentlichen Grund für ihr Engagement. Hohe Zustimmung erlangte ferner die Motivation zur Mitgestaltung von Gesellschaft. Schließlich wollten über $90 \%$ der Befragten auch ein Zeichen gegen Rassismus setzen. Die Zustimmung zu dem Ziel, ein Zeichen gegen die konkrete Flüchtlingspolitik setzen zu wollen, fiel demgegenüber aber deutlich geringer aus (ebd., S. 32f.). Für Österreich liegen derzeit keine repräsentativen Forschungen zum freiwilligen Engagement im Bereich Flucht/Migration vor. Kleinere regionale Befragungen erbringen in Bezug auf soziodemographische Merkmale der Freiwilligen ähnliche Ergebnisse wie die zitierten deutschen Studien (Sundl 2015).

\subsection{Medialer und politischer Diskurs}

Zweifelsohne trug eine extensive mediale Berichterstattung über die im Spätsommer 2015 in Deutschland und Österreich ankommenden Schutzsuchenden wesentlich zur raschen und umfangreichen Mobilisierung von Freiwilligen bei. Aber auch das Phänomen der Mobilisierung selbst war eine Zeit lang unter dem Schlagwort der sogenannten Willkommenskultur Gegenstand medialer Darstellungen. Um die Motive, Erfahrungen und (politischen) Lernprozesse der freiwillig Engagierten zu analysieren, ist ein Blick auf den öffentlichen Diskurs daher unverzichtbar. Dieser wird trotz zunehmender Bedeutung sozialer Medien nach wie vor maßgeblich durch bestimmte Leitmedien wie marktführende Zeitungen, Zeitschriften und Rundfunkanstalten geprägt (Vollmer und Karakayali 2017, S. 2) - deren Impulse im Übrigen wiederum von sozialen Medien aufgegriffen werden. Eine Medienanalyse von acht in Deutschland erscheinenden Printmedien (Zeitraum März 2015 bis März 2016), die durch Experteninterviews ergänzt wurde, zeigt beispielsweise auf, mit welchen

\footnotetext{
${ }^{2}$ Ergebnisse einer dritten Befragung 2016 waren bis dato noch nicht publiziert.
} 
Bedingungen die Akzeptanz Geflüchteter im Diskurs verknüpft wird. So lässt sich etwa eine positive Darstellung jener Menschen, die Hilfe verdienen, in Zeitungen ebenso wie in Befragungen von Freiwilligen wiederfinden. Hilfe zu verdienen resultiert beispielsweise am häufigsten aus der Vorstellung, dass die Betroffenen zur Migration gezwungen wurden (,echte Flüchtlinge“). Dies wird durch das Narrativ von Familien und Kindern in weit höherem Ausmaß repräsentiert als etwa im Bild junger Männer, die eher als ökonomisch motivierte Migranten konnotiert sind (ebd., S. 3). Zusätzlich wird hier oftmals auf eine Idee von „Unschuld“, wie sie etwa mit dem Bild des toten Kindes Aylan Kurdi vermittelt wurde, rekurriert (ebd. S. 8). Wird diese handlungsleitende Idee jedoch - beispielsweise durch eine als nicht ausreichend empfundene Dankbarkeit der Hilfeempfangenden - enttäuscht, kann dies zu starker Frustration bei den Unterstützenden führen.

Die Situation des Sommers 2015 war geeignet, eine emotionalisierte mediale Darstellung eines außergewöhnlichen „Katastrophenzustandes“ zu schaffen, die schließlich eine breite Welle an Hilfsbereitschaft nach sich zog. Diese wurde wiederum durch einschlägige Unterstützungserklärungen von Persönlichkeiten und diversen Institutionen verstärkt. Die massenhafte Hilfsbereitschaft vieler Bürgerinnen und Bürger wurde eine Zeit lang in fast allen Medien ausführlich gewürdigt. Hier drückte sich oftmals eine Art nationalen Stolzes über die Warmherzigkeit und Hilfsbereitschaft der Deutschen (ebd., S. 10) aus. Inwieweit in diesem Zusammenhang Emotionen auch aus politischem Kalkül gezielt gesteuert wurden, wäre einer weiteren Analyse wert.

Die Berichterstattung über die Fluchtbewegungen 2015 liest sich über einen relativ langen Zeitraum durchwegs wohlwollend, entwickelte sich dann jedoch stärker in Richtung kritischer und ablehnender Beiträge - dies war für Deutschland (vgl. ebd.) ebenso beobachtbar wie in Österreich im Zeitraum der Durchführung unserer empirischen Studie. Ohne einen klaren Zeitpunkt dieser Verschiebung ausmachen zu können, zeigen sich doch kritische Ereignisse im Diskurs - beispielsweise durch die Terroranschläge von Paris oder in besonderem Ausmaß nach den Übergriffen in der Kölner Silvesternacht 2015/16. Einschlägige Berichte knüpften in mehrerlei Hinsicht an bereits zuvor präsenten Sicherheitsdiskursen sowie an den oben erwähnten Narrativen über die fragile Definition einer vermeintlichen Hilfswürdigkeit Geflüchteter an. ,The notion or the concept of the young male adult ranges at the opposite end of the vulnerability-deservingness scale and more easily fulfills the requirements of a discursive pattern in which migrants are framed as evil-doers" (ebd., S. 13). Nach diesem Ereignis ließ sich ein massiver Anstieg negativer und feindseliger Debatten in diversen Medien konstatieren bzw. drückte sich ein Stimmungsumschwung in konkreten Protestaktionen, etwa gegen die Unterbringung Geflüchteter, aus. Wie diese Ereignisse auch von freiwillig Engagierten rezipiert wurden, wird an einer späteren Stelle erneut aufgegriffen. 


\section{Politische Lern- und Bildungsprozesse}

Das demokratiepolitische Potenzial von freiwilligem Engagement wurde ebenso wie andere (zumeist informelle) Lern- und Bildungsprozesse bereits öfters analysiert (Duguid et al. 2013; Trumann 2013; Düx et al. 2009; Foley 1999) - selbst wenn noch vor rund zehn Jahren informelles politisches Lernen als wenig erforscht galt (Schugurensky 2006, S. 163). Diese Studien zeigen, dass Freiwillige ihr soziales und politisches Bewusstsein durch Verantwortungsübernahme und intensive Konfrontation mit gesellschaftspolitischen Bedingungen potenziell weiterentwickeln. Die individuellen und kollektiven Lernprozesse im Engagement können als „Türöffner“ für politische Partizipation fungieren (Trumann 2013, S. 272).

\subsection{Lernkontexte und -strategien}

Die empirischen Ergebnisse zu Lernprozessen Freiwilliger im Bereich Flucht/ Migration aus unserer in Österreich durchgeführten explorativen Studie decken sich weitgehend mit anderen Forschungsarbeiten, die rund um die aktuellen Flüchtlingsbewegungen in Deutschland publiziert wurden. Unsere Daten machen deutlich, dass große Teile der Unterstützungsarbeit auch abseits etablierter Wohlfahrtsverbände geleistet wurden und somit nicht auf vorhandenen Strukturen aufsetzen konnten. Viele Gruppen entwickelten schrittweise partizipative Verfahren und etablierten notwendige Regeln und Rollenverteilungen, um handlungsfähig zu sein. Ferner eignete man sich etwa asylrechtlich relevantes Wissen mittels individueller als auch kollektiv organisierter Lernstrategien an, wobei die Wissensweitergabe und Planungen in hohem Ausmaß über Social-Media erfolgten. Ähnlich beschreiben Trumann (2013) und Foley (1999), dass in zivilgesellschaftlichen Gruppen nicht nur voneinander gelernt wird, indem man bereits vorhandenes Wissen austauscht, sondern auch neues Wissen in die Gruppe eingespeist wird.

\subsection{Kritisches Verständnis politischer Zusammenhänge und Ausweitung des Aktionsradius}

Bürgerschaftliche Initiativen erweitern fallweise ihren Aktionsradius über das je konkrete Probleme hinaus, da sie oftmals während ihrer Tätigkeit lernen, ihr Thema in einen weiteren politischen Kontext zu stellen und sich dann häufig auch für diesen in gewisser Weise verantwortlich fühlen (vgl. Trumann 2013). In unseren Interviews wurde etwa berichtet, dass man sich im Angesicht der prekären Lebenslagen von Schutzsuchenden sowie deren Berichte aus den Herkunftsländern veranlasst fühle, über das eigene privilegierte Leben nachzudenken und daraus auch eine Verantwortung zur solidarischen Hilfe abzuleiten. Ähnliche Ergebnisse erbringt die Studie von Jungk und Morrin (2017). Die unmittelbare Erfahrung der Restriktionen, denen Geflüchtete ausgesetzt sind, trug bei einigen Freiwilligen zur Entfaltung eines kritischen Verständnisses über Migrationsregime sowie dahinter liegender globaler Zusammenhänge bei. Das soziale Engagement entfaltet somit potenziell transformative Wirkungen (vgl. auch Braun 2017; Fleischmann und Steinhilper 2017). Um mit solchen Erkenntnissen einen Umgang zu finden, aber auch um sich feindseligen, 
etwa rechtsextremistisch motivierten Angriffen zu widersetzen, mussten spezifische Handlungspraxen erarbeitet werden. So wurde von manchen Gruppen beispielsweise die unmittelbare Unterstützungsarbeit um Aktivitäten, wie die Sensibilisierung der kommunalen Bevölkerung oder auch die Organisation von Protesten gegen Abschiebungen u.ä., erweitert. Es wurden hier also in einigen Fällen prononciertere und kritische Positionen zum politischen und sozialen Umgang mit Geflüchteten entwickelt sowie in konkrete Handlungen, die sich an eine breitere Öffentlichkeit richteten, übersetzt.

\subsection{Reflexion eigener Wertvorstellungen und Vorurteile}

Unsere Erhebung zeigte, dass u.a. die persönlich erlebten und positiv bewerteten oft als ,interkulturell“ bezeichneten Lernerfahrungen im Kontakt mit Geflüchteten (vgl. ebenso Jungk und Morrin 2017, S. 45) - einschließlich der Reflexion eigener Ängste/Vorurteile - Gegenstand erweiterter Bildungsaktivitäten wurden, welche in Folge einem größeren Adressatenkreis offen standen. In weit geringerem Umfang wurden hingegen Selbstvertretungsansprüche Geflüchteter (Refugee Protest Camp) unterstützt.

Schließlich ist ein weiterer Lernimpuls im sozialen Druck zu sehen, dem viele Freiwillige seitens der Öffentlichkeit, aber auch ihres privaten und beruflichen Umfeldes ausgesetzt waren. Sie sahen sich gezwungen, ihr Engagement permanent rechtfertigen zu müssen. Dafür waren stringente argumentative Strategien zu entwickeln, was neben Wissen und Analyse auch eine Reflexion der eigenen Wertvorstellungen und deren Begründung voraussetzt. Diese wurden zum Teil im Diskurs mit anderen Aktivisten und Aktivistinnen (u.a. via Social Media) erarbeitet. Dass viele freiwillig Engagierte differenzierte und keinesfalls idealisierte Vorstellungen über die Situation entfalten, zeigen Jungk und Morrin (2017, S. 77 ff.).

\subsection{Ausstieg aus dem Engagement und politisches Selbstverständnis}

Ein rassistischer und feindseliger öffentlicher Diskurs muss allerdings nicht zwangsläufig zur Herausbildung eines prononcierten Solidaritätsverständnisses oder gar widerständiger politischer Praxen bei den freiwillig Engagierten führen. Viele Helfende scheinen, so legen es unsere empirischen Erkenntnisse nahe, durch negative Medienberichte durchaus auch verunsichert worden zu sein und sind teilweise gänzlich aus dem Engagement ausgestiegen. Selbst in Österreich markiert die mediale Berichterstattung über die Kölner Silvesternacht gewissermaßen eine Zäsur und führte bei manchen Engagierten zu einer Generalisierung negativer Eindrücke auf alle „Flüchtlinge“ oder „,muslimische Migrantinnen und Migranten“. Studien aus Deutschland haben diesen Effekt zwar ebenfalls festgestellt, zugleich aber auch in einigen Regionen einen konstanten Zustrom neuer Freiwilliger konstatiert. Hamann et al. (2016, S. 44f.) haben in einigen befragten Kommunen beobachtet, dass Proteste gegen Asylwerbende sogar freiwilliges Engagement lokal verstärken konnten.

Oftmals stehen hinter dem Abbruch des Engagements diverse weitere Gründe wie physische und psychische Überlastung, aber auch enttäuschende Erfahrungen im individuellen Kontakt. Sie führen in manchen Fällen zu einem Umschlagen positiver 
Haltungen in Ressentiments. Dies mag u. a. mit einem Grundproblem der asymmetrischen Konstellation „humanitären“ Engagements einhergehen, wonach hier häufig vor allem eine Idee von charity verfolgt wird, während demgegenüber die Durchsetzung von Rechten oder das Empowerment Geflüchteter weniger oder gar nicht im Mittelpunkt des Engagements stehen.

„The dominant ethos of humanitarianism is charity, and charitable giving is carried out by persons voluntarily engaged in giving to those in need. (...) Thus in the context of giving humanitarian assistance, whether or not they are aware of it, humanitarian workers stand in an asymmetrical relationship to refugees who are symbolically disempowered through becoming clients of those upon whom they are dependent for the means of survival and security." (Harrell-Bond 2002, S. 55).

Inwieweit freiwillig Tätige ihren Einsatz selbst als politische Arbeit betrachten, ist durchaus unterschiedlich - manche grenzen sich von einer solchen Selbstdefinition ganz ab und verstehen sich als lediglich humanitär Engagierte, andere wiederum wollen ihr Tun als gesellschaftsverändernden Beitrag verstanden wissen (Jungk und Morrin 2017; Fleischmann und Steinhilper 2017). Wir sehen in unserem empirischen Material Beispiele sowohl dafür, dass ein bereits vorhandenes politisches Selbstverständnis als Motivationsfaktor freiwilliges Engagement bedingt, als auch dafür, dass ein einschlägiges Selbstverständnis erst im Laufe des Engagements entwickelt wird.

Die Frage, in welcher Weise unterschiedliche Faktoren, wie z. B. Vorwissen, biografische Erfahrungen, ein spezifisches politisches Selbstverständnis oder auch begleitende Bildungsangebote die Bewältigung schwieriger und verunsichernder Situationen im Engagement beeinflussen, lässt sich anhand der uns bislang vorliegenden Daten noch nicht eindeutig beantworten. Zweifelsohne sind freiwillig Engagierte in ihrer Tätigkeit oftmals mit irritierenden und ambivalenten Herausforderungen (wie Wertekonflikten) konfrontiert, die Frustrationen auslösen und von den Freiwilligen reflexiv bearbeitet werden müssen (Jungk und Morrin 2017, S. 63 ff.). Folglich ist auch die Frage interessant, inwiefern dafür Ressourcen individueller und kollektiver Art - wie z. B. (selbst-)organisierte Bildungsräume - zur Verfügung stehen.

\section{Freiwilliges Engagement als Ausdruck aktiver (und inklusiver?) Bürgerschaft}

Für freiwillig Engagierte bedeutet die Mehrdimensionalität von Fluchtmigration (soziodemografische Zusammensetzung der Geflüchteten, Migrationsgründe, unterschiedlicher rechtlicher Status) sowie des polarisierten medialen und politischen Diskurses die Notwendigkeit, sich mit ihrer eigenen Verantwortlichkeit der Gesellschaft gegenüber auseinanderzusetzen. Durch ihr soziales Engagement üben Freiwillige eine aktive Bürgerschaft aus und leisten dadurch einen wichtigen Beitrag zur Sozialintegration (vgl. Himmelmann 2018, S. 37), aber beispielsweise auch zum Zurückdrängen rechtsextremer Tendenzen (Hamann et al. 2016, S. 14). 


\subsection{Die Rolle von freiwilligem Engagement in aktiver Bürgerschaft}

Die Bedeutung aktiver Bürgerschaft ${ }^{3}$ ist von den diversen (nationalstaatlichen) Kontexten und demokratischen Traditionen abhängig (Kennedy 2007) und wird dementsprechend oft unterschiedlich bestimmt. Hilfreich ist Alistair Ross' (2012) Definition von active citizens (aktiven Bürgerinnen/Bürgern) als jene, die sich kritisch in gesellschaftliche Ereignisse einbringen, Gesetze auf Ungerechtigkeiten und Veränderungsmöglichkeiten hin prüfen und das soziale Geschehen beeinflussen möchten (ebd. 7f). Ross bezieht sich ebenso wie Julie Nelson und David Kerr (2006) auf eine von Kerry J. Kennedy (2006) eingeführte Ausdifferenzierung von vier Ebenen von aktiver Bürgerschaft. Dies sind (1) conventional citizenship: die Partizipation in konventionellen politischen Aktivitäten wie zu wählen, einer politischen Partei anzugehören oder für ein politisches Amt zu kandidieren, (2) social movement citizenship: das Engagement in freiwilligen Aktivitäten wie Freiwilligenarbeit in Vereinen und Geldsammlungen (social movement citizenship), (3) social change citizenship: Aktivitäten, die politische und soziale Veränderungen herbeiführen wollen, sei es durch legale Mittel wie Briefe versenden und Petitionen unterschreiben, oder sei es mittels illegaler Mittel wie Verkehrsblockaden oder Gebäudebesetzungen, und (4) economic/enterprise citizenship, bei welcher auf ein individuelles Bürgerschaftskonzept Bezug genommen wird und finanzielle Unabhängigkeit und unternehmerische Werte hochgehalten werden.

Unter aktiver Bürgerschaft kann prinzipiell jede dieser vier Ebenen verstanden werden - auch Benedikt Widmaier (2011, S. 50) sieht es als international anerkannt an, dass aktive Bürgerschaft sowohl traditionelle Formen wie die Beteiligung an Wahlen und Mitgliedschaften in Parteien, Aktivitäten in Nichtregierungsorganisationen, als auch themenbezogenes politisches Engagement beinhaltet. Oftmals wird mit aktiver Bürgerschaft vom drittgenannten Verständnis, dem social change citizenship, ausgegangen, so wie dies beispielsweise die meisten Autorinnen und Autoren im Special Issue des International Journal of Progressive Education machen (Ross 2012).

In einer breiten Definition von aktiver Bürgerschaft wäre jede Form des freiwilligen Engagements in Solidarität mit Geflüchteten eine Wahrnehmung aktiver Bürgerschaft. In Bezug auf Lern- und Bildungsprozesse können wir bei einigen der Interviewpartnerinnen und Interviewpartner aber eine Entwicklung hin zu einer aktiven Bürgerschaft spezifisch im Sinne einer social change citizenship feststellen: Es werden kritische Positionierungen erarbeitet, sowie Aktivitäten zur öffentlichen Bewusstseinsbildung und politische Proteste durchgeführt.

Als grundlegend für Partizipation im Rahmen von aktiver Bürgerschaft werden gegenseitige Achtung und Gewaltlosigkeit, sowie Menschenrechte und Demokratie angesehen (Widmaier 2011, S. 50). Insofern ist es zusätzlich lohnenswert, das freiwillige Engagement für Geflüchtete unter der Forschungsperspektive der Inclusive Citizenship zu untersuchen.

\footnotetext{
${ }^{3}$ Wir verwenden hier den deutschen Begriff äquivalent zum englischen ,active citizenship“.
} 


\subsection{Inclusive Citizenship lernen?}

Unter der Perspektive der Inclusive Citizenship werden die gesellschaftlichen Konstruktionen von Citizenship und die damit einhergehenden Prozesse der Exklusion bestimmter Bevölkerungsgruppen in den Blick genommen und nach bestehenden oder anzustrebenden Praxen der Inklusion gefragt (Kleinschmidt und Lange 2018). Die Forschungsperspektive Inclusive Citizenship untersucht v. a. Phänomene der Migrationsgesellschaft, des Rassismus, der Gender(un)gerechtigkeit, des gesellschaftlichen Umgangs mit Behinderung sowie die Verhandlung von Armut in Geschichte und Gegenwart. Das normative Ziel dabei ist eine inklusive, demokratische Gesellschaft (ebd.). Für migrationsgesellschaftliche Fragestellungen ist relevant, dass das Konzept der Inclusive Citizenship auch globale Dimensionen in die Analyse einbezieht, Strategien anderer Weltregionen erforscht und so versucht, einen ,methodologischen Nationalismus“ (Wimmer und Glick Schiller 2003) zu überwinden. Dies entspricht jenen Vorstellungen von aktiver Bürgerschaft, welche über die Grenzen des Nationalstaates blicken. ${ }^{4}$

Entsprechend der Perspektive der Inclusive Citizenship möchten wir abschlieBend die beschriebenen Bildungsprozesse auf die Frage hin analysieren, inwiefern das Engagement für Geflüchtete dazu beiträgt, dass die Freiwilligen ein besseres Verständnis oder sogar Praxis von Inclusive Citizenship entwickeln. Jede Bürgerin und jeder Bürger entscheidet selbst (bzw. unter den jeweiligen sozialen und biographischen Bedingungen), wie ihre oder seine Citizenship gestaltet sein soll und auf welcher Ebene eine gesellschaftliche Involvierung erfolgt. Wie beschrieben, reicht dies von reiner Passivität über die Beteiligung an Wahlen bis hin zu sozialem Engagement oder (partei)politischer Aktivität. Die in der Unterstützung von Geflüchteten freiwillig Tätigen übernehmen ganz deutlich eine aktive, die Gesellschaft gestaltende Rolle.

In der Realisierung ihrer aktiven Bürgerschaft entsprechen nicht alle Handlungen einem inklusiven Ansatz. Viele Geflüchtete, Migrantinnen und Migranten, die selbst als Freiwillige tätig sind, machen die Erfahrung, dass sie keine gleichwertigen Partizipationsmöglichkeiten in der Community der Freiwilligen haben und ihr Wissen nicht adäquat anerkannt wird (vgl. Braun 2017). Paternalistische Tendenzen sind nicht nur auf der Achse der Helfenden gegenüber Unterstützten festzustellen, sondern auch in Machtverhältnissen zwischen Weißen ${ }^{5}$ und Nicht-Weißen Aktivistinnen und Aktivisten (vgl. Castro Varela und Heinemann 2016; Fleischmann und Steinhilper 2017; van Dyk und Misbach 2016). Gleichzeitig aber ermöglicht der gemeinsame Aktivismus auch, dass die Weißen Engagierten von ihren Kolleginnen und Kollegen mit Flucht- bzw. Migrationserfahrung durchaus viel lernen, etwa in Hinblick auf Rassismuskritik und/oder adäquate Planung der Unterstützungsangebote (Braun 2017, S. 45).

\footnotetext{
${ }^{4}$ Vgl. hierzu auch die Diskussion um Global Citizenship (Ross 2012, S. 9) und Denizenship (Benton 2010; Golash-Boza 2016).

5 Die Großschreibung verweist auf die Kategorisierung entsprechend der sozialen Positionierung und Zuschreibung.
} 
Den Ansatz der Inclusive Citizenship zu verfolgen würde bedeuten, den Prozess in Hinblick auf diese sowie andere Machtverhältnisse, wie etwa Klasse und Geschlecht, möglichst demokratisch zu gestalten. Die Freiwilligen müssten ihrer Heterogenität Rechnung tragen und auch für Nicht-Weiße gleichberechtigte Zugänge, Mitsprachemöglichkeiten und Repräsentation im Außenauftritt herstellen. Gleichzeitig halten wir eine kritische Reflexion von paternalistischen und viktimisierenden Zugängen zum Engagement für grundlegend.

\section{Konklusion}

Wenn von freiwilligem Engagement als active Citizenship gesprochen wird und dabei der Fokus auf politischen Bildungsprozessen liegt, knüpft dies unweigerlich an den Diskurs zu Citizenship Education (oder „Demokratiebildung“) an. Hinsichtlich der Lernprozesse in bürgerschaftlichen Initiativen für Geflüchtete fällt vor allem, wie dargestellt, das Lernen über Migrations- und Integrationspolitik auf. Migrationsregime kritisch zu reflektieren kann bedeuten, Ein- und Ausschlüsse der (Staats-)Bürgerschaft selbst zu hinterfragen - wie es einer inklusiven Perspektive entspräche. Inwieweit ein solcher Bildungsprozess durch freiwilliges Engagement angestoßen wird bzw. welche anderen Faktoren wirksam werden, ist eine noch zu erforschende Frage.

Die bei vielen Freiwilligen festgestellte Infragestellung von Herrschafts- und Machtstrukturen, die Bildung eines kritisch-reflexiven Urteils und das Kennenlernen möglicher Handlungsstrategien sind wichtige Ziele von Citizenship Education (Kenner und Lange 2018, S. 9). Citizenship Education heißt daher auch, Einflussmöglichkeiten auf Entscheidungsprozesse durch die Zivilgesellschaft kennenzulernen und zu erproben (ebd., S. 11). Unsere Beobachtungen zeigen, dass jene Freiwilligen, die ein politisches Verständnis entwickeln, sich zum Teil ebenso um politische Veränderungen bemühen. $\mathrm{Ob}$ eine Desillusionierung durch stagnierende oder als negativ wahrgenommene politische Entwicklungen einen weiteren Grund für den Abbruch des Engagements darstellen könnte, ist erst durch weitere empirische Studien (u.a. im Rahmen unseres aktuellen Forschungsprojektes) zu klären.

Insgesamt ist festzustellen, dass viele Freiwillige in diesem Feld im Zuge der Lern- und Bildungsprozesse neue oder transformierte Bedeutungsschemata entwickeln. Inwiefern dies auch für jene Menschen der Fall ist, welche ihr Engagement beendet haben, wird unsere aktuelle Studie noch näher beleuchten.

Open Access Dieser Artikel wird unter der Creative Commons Namensnennung 4.0 International Lizenz (http://creativecommons.org/licenses/by/4.0/deed.de) veröffentlicht, welche die Nutzung, Vervielfältigung, Bearbeitung, Verbreitung und Wiedergabe in jeglichem Medium und Format erlaubt, sofern Sie den/die ursprünglichen Autor(en) und die Quelle ordnungsgemäß nennen, einen Link zur Creative Commons Lizenz beifügen und angeben, ob Änderungen vorgenommen wurden.

\section{Literatur}

Ahrens, P.-A. (2017). Skepsis und Zuversicht - Wie blickt Deutschland auf Flüchtlinge? https://www.siekd.de/images/Fluechtlingsstudie_SP_PW_final.pdf. Zugegriffen: 13. März 2018. 
BAMF - Bundesamt für Migration und Flüchtlinge (2017). Das Bundesamt in Zahlen 2016. Asyl, Migration und Integration. http://www.bamf.de/SharedDocs/Anlagen/DE/Publikationen/Broschueren/ bundesamt-in-zahlen-2016.pdf?_blob=publicationFile. Zugegriffen: 13. März 2018.

Benton, M. (2010). A theory of Denizenship. http://discovery.ucl.ac.uk/624490/1/624490.pdf. Zugegriffen: 7. März 2018.

BMI - Bundesministerium für Inneres (2017). Asylstatistik 2016. http://www.bmi.gv.at/301/Statistiken/ files/Jahresstatistiken/Jahresstatistik_Asyl_2016.pdf. Zugegriffen: 13. März 2018.

Braun, K. (2017). Decolonial perspectives on charitable spaces of „welcome culture“ in Germany. Social Inclusion, 5(3), 38-48.

Castro Varela, M., \& Heinemann, A. (2016). Mitleid, Paternalismus, Solidarität. Zur Rolle von Affekten in der politisch-kulturellen Arbeit. In C. Gritschke \& M. Ziese (Hrsg.), Geflüchtete und Kulturelle Bildung (S. 51-66). Bielefeld: transcript.

Duguid, F., Mündel, C., \& Schugurensky, D. (2013). Volunteer work, informal learning and social action. Rotterdam: Sense Publishers.

Düx, W., Prein, G., Sass, E., \& Tully, C. (2009). Kompetenzerwerb im freiwilligen Engagement. Wiesbaden: Springer VS.

van Dyk, S., \& Misbach, E. (2016). Zur politischen Ökonomie des Helfens. Flüchtlingspolitik und Engagement im flexiblen Kapitalismus. PROKLA, 183, 46(2), 205-227.

Eurostat (2017). Statistiken über Asyl. http://ec.europa.eu/eurostat/statistics-explained/index.php/Asylum_ statistics/de. Zugegriffen: 13. März 2018.

Fleischmann, L., \& Steinhilper, E. (2017). The myth of apolitical volunteering for refugees. German welcome culture and a new dispositif of helping. Social Inclusion, 5(3), 17-27.

Foley, G. (1999). Learning in social action. A contribution to understanding informal education. New York: Zed Books.

Fonds Soziales Wien (2017). Flüchtlinge, Asyl und Grundversorgung. Grafiken und Daten zu Wien, Österreich und EU. https://www.fsw.at/downloads/satzung_berichte/FSWFaktenFluechtlinge-1.pdf. Zugegriffen: 13. März 2018.

Golash-Boza, T. (2016). Feeling like a citizen, living as a denizen: deportees sense of belonging. American Behavioral Scientist, 60(13), 1575-1589.

Hamann, U., Karakayali, S., Wallis, M., \& Höfler, L. J. (2016). Koordinationsmodelle und Herausforderungen ehrenamtlicher Flüchtlingshilfe in den Kommunen. https://www.bertelsmann-stiftung.de/ fileadmin/files/BSt/Publikationen/GrauePublikationen/Koordinationsmodelle_und_

Herausforderungen_ehrenamtlicher_Fluechtlingshilfe_in_den_Kommunen.pdf. Zugegriffen: 13. März 2018. Qualitative Studie des Berliner Instituts für empirische Integrations- und Migrationsforschung.

Harrell-Bond, B. (2002). Can humanitarian work with refugees be humane? Human Rights Quarterly, 24, 51-85.

Himmelmann, G. (2018). Demokratie als Gesellschaftsform - Politische Bildung und Zivilgesellschaft. In S. Kenner \& D. Lange (Hrsg.), Citizenship Education. Konzepte, Anregungen und Ideen zur Demokratiebildung (S. 27-38). Frankfurt/Main: Wochenschauverlag.

Hofer, B. (2015). Bericht zur Lage und zu den Perspektiven des Freiwilligen Engagements in Österreich. 2. Freiwilligenbericht. http://www.der-paritaetische.de/fileadmin/user_upload/Publikationen/ doc/2017-12-20_ehrenamt-STUDIE.pdf. Zugegriffen: 13. März 2018.

Jungk, S., \& Morrin, S. (2017). Ehrenamt mit Geflüchteten. Ein Praxisforschungsprojekt über die Ressourcen, Erwartungen und Erfahrungen von ehrenamtlichen Helfer_innen in pädagogischen Settings. http://www.der-paritaetische.de/fileadmin/user_upload/Publikationen/doc/2017-12-20_ehrenamtSTUDIE.pdf. Zugegriffen: 16. März 2018. Abschlussbericht.

Karakayali, S., \& Kleist, O.J. (2016). EFA-Studie 2. Strukturen und Motive der ehrenamtlichen Flüchtlingsarbeit in Deutschland. Forschungsbericht. http://www.fluechtlingsrat-brandenburg.de/wpcontent/uploads/2016/08/Studie_EFA2_BIM_11082016_VOE.pdf. Zugegriffen: 13. März 2018. Ergebnisse einer explorativen Umfrage von November/Dezember 2015.

Kennedy, J. K. (2006). Towards a conceptual framework for understanding active and passive citizenship. Unpublished report

Kennedy, J. K. (2007). Student constructions of ,active citizenship“: What does participation mean to students? British Journal of Educational Studies, 55(3), 304-324. https://doi.org/10.1111/j.1467-8527. 2007.00381.x.

Kenner, S., \& Lange, D. (2018). Einführung: Citizenship Education. In S. Kenner \& D. Lange (Hrsg.), Citizenship Education. Konzepte, Anregungen und Ideen zur Demokratiebildung (S. 9-17). Frankfurt/ Main: Wochenschau Verlag. 
Kleinschmidt, M., \& Lange, D. (2018). Inclusive Citizenship. Exklusion und Praxen inklusiver Bürgerschaft als Ausgangspunkt einer emanzipatorischen politischen Bildung. In M. Kleinschmidt \& D. Lange (Hrsg.), Emanzipation: Zum Konzept der Mündigkeit in der politischen Bildung (S. 96-107). Frankfurt/Main: Wochenschauverlag.

Koller, H.-C. (2012). Bildung anders denken. Einführung in die Theorie transformatorischer Bildungsprozesse. Stuttgart: Kohlhammer.

Nelson, J., \& Kerr, D. (2006). Active citizenship in INCA countries: definitions, policies, practices and outcomes: final report. https://www.nfer.ac.uk/publications/QAC02/QAC02.pdf. Zugegriffen: 27. Juni 2018.

Ross, A. (2012). Education for active citizenship: practices, policies, promises. International Journal of Progressive Education, 8(3), 7-14.

Schugurensky, D. (2006). „This is our school of citizenship“: informal learning in local democracy. Counterpoints, 249, 163-182.

Simonson, J., Vogel, C., \& Tesch-Römer, C. (2014). Freiwilliges Engagement in Deutschland. Der deutsche Freiwilligensurvey 2014. Wiesbaden: Springer VS.

Sundl, B. (2015). Jahresbericht der Servicestelle freiwilliges Engagement. https://www.caritas-steiermark. at/fileadmin/storage/steiermark/documents/Spenden-und-Helfen/Freiwillige-Mitarbeit/JB15-web. pdf. Zugegriffen: 13. März 2018.

Trumann, J. (2013). Lernen in Bewegung(en). Bielefeld: transcript.

UNHCR (2017). Global Trends. Forced Displacement in 2016. http://www.unher.org/5943e8a34.pdf. Zugegriffen: 13. März 2018.

Ünsal, N. (2015). Challenging 'refugees' and 'supporters'. Intersectional power structures in the refugee movement in berlin. http://movements-journal.org/issues/02.kaempfe/09.ünsal--refugees-supportersoplatz-intersectionality.pdf. Zugegriffen: 13. März 2018.

Vollmer, B., \& Karakayali, S. (2017). The volatility of the discourse on refugees in Germany. Journal of Immigrant \& Refugee Studies. https://doi.org/10.1080/15562948.2017.1288284.

Widmaier, B. (2011). Lassen sich aktive Bürgerschaft und Bürgerschaftliche Kompetenzen messen? Europäische Planungsdaten für Lebenslanges Lernen und Politische Bildung. In B. Widmaier \& F. Nonnenmacher (Hrsg.), Active Citizenship Education. Internationale Anstöße für die Politische Bildung (S. 45-64). Schwalbach/Ts.: Wochenschau-Verlag.

Wimmer, A., \& Schiller Glick, N. (2003). Methodological nationalism, the social sciences, and the study of migration. An essay in historical epistemology. International Migration Review, 3, 576-610.

Zimmer, A., \& Vilain, M. (2005). Bürgerschaftliches Engagement heute. Ibbenbüren: Vereinsdruckerei. 